\title{
Wound Myiasis Caused by Sarcophaga (Liopygia) Argyrostoma (Robineau-Desvoidy) (Diptera: Sarcophagidae): Additional Evidences of the Morphological Identification Dilemma and Molecular Investigation
}

\author{
Annunziata Giangaspero, ${ }^{1}$ Marianna Marangi, ${ }^{1}$ Antonio Balotta, ${ }^{2}$ Claudio Venturelli, ${ }^{3}$ \\ Krzysztof Szpila, ${ }^{4}$ and Antonella Di Palma ${ }^{1}$ \\ ${ }^{1}$ Dipartimento di Scienze Agrarie, degli Alimenti e dell'Ambiente, Università degli Studi di Foggia, Via Napoli 25, Foggia, Italy \\ ${ }^{2}$ Unità Operativa di Geriatria, Ospedale M. Bufalini, AUSL della Romagna, Ravenna, Italy \\ ${ }^{3}$ Dipartimento di Sanità Pubblica, AUSL della Romagna, Ravenna, Italy \\ ${ }^{4}$ Chair of Ecology and Biogeography, Faculty of Biology and Environmental Protection, Nicolaus Copernicus University, \\ Lwowska 1, 87-100 Toruń, Poland
}

Correspondence should be addressed to Annunziata Giangaspero; annunziata.giangaspero@unifg.it

Received 13 October 2016; Accepted 8 December 2016; Published 29 January 2017

Academic Editor: Gabriella Cancrini

Copyright (C) 2017 Annunziata Giangaspero et al. This is an open access article distributed under the Creative Commons Attribution License, which permits unrestricted use, distribution, and reproduction in any medium, provided the original work is properly cited.

\begin{abstract}
In Mediterranean countries, Sarcophaga (Liopygia) crassipalpis, Sarcophaga (L.) argyrostoma, and Sarcophaga (L.) cultellata share the same ecological niche and can be responsible of myiasis. In this study, the main morphological characters of a larva found in a hospitalized woman were described and illustrated by light and SEM microscopy and the features discussed. Then, a fragment within the mitochondrial encoded cytochrome c oxidase subunit I (coxI) gene of $\sim 735 \mathrm{bp}$ was amplified and sequenced. The molecular investigation was necessary to confirm the species Sarcophaga (Liopygia) argyrostoma (99\% of identity). Our findings showed that morphological descriptions of larvae of three Mediterranean species of Liopygia available in several papers might not be clear enough to allow for comparison and correct identification. Until results of reliable comparative studies of larvae of all three species will be available, the use of molecular tools is crucial, to avoid misleading or incomplete identification, and in particular when a myiasis becomes a legal issue.
\end{abstract}

\section{Introduction}

In Europe, 33 Sarcophaga Meigen species attracted to large carrion have been described [1]. Several of them pose confirmed status of facultative myiasis agents [2]. Among them, in the Mediterranean countries, three Sarcophaga species belonging to the same subgenus (Liopygia) share the same ecological niche: Sarcophaga (Liopygia) crassipalpis Macquart, 1839, Sarcophaga (L.) argyrostoma Robineau-Desvoidy, 1830, and Sarcophaga (L.) cultellata Pandelle, 1986 [3]. These species are included in the category of "nosocomial myiasis agents" because they have been found in wound myiasis affecting hospital patients who are immobilized, often old, and poorly cared [4-8]. Flies are attracted by the smell of fresh or infected and untreated wounds and lay eggs (or larvae as in the case of Sarcophaga), which then develop in necrotic or injured tissues and sometimes cause or exacerbate a wound [9]. This can often become a legal problem, which entails an urgent need to identify the larvae.

Where the geographical species distribution overlaps, as happens for these three Sarcophaga species in the Mediterranean area [3], and only one single larva is available, differentiation of these three species becomes very challenging $[3,10]$. Thus, cross-checking of the morphological and molecular data is highly recommended for both adult [11] and larval specimens [10].

The mitochondrial encoded cytochrome c oxidase subunit I (coxI) gene has been shown to be a major candidate 
gene for molecular identification of forensic flies [12] and is currently the most widely used genetic marker for differentiation of Sarcophaga species of forensic interest thanks to its intraspecific (below 1.5\%) and the interspecific divergence (2$2.5 \%)[13,14]$.

The aim of this contribution is to report a case of nosocomial myiasis, to describe and illustrate the main morphological characters used in identification of the collected larva, compared to those available in the literature, and to highlight the need for molecular support for correct identification.

\section{Materials and Methods}

In September 2014, a 101-year-old female patient who had been bedridden for months in a geriatric home in Cesena was specifically admitted to Bufalini Hospital with a "parasitic infestation" of a wound. A single larva was found on the wound, removed with pincers, and preserved in ethanol $($ ETOH $80 \% \mathrm{v} / \mathrm{v})$. The wound was then disinfected using chlorhexidine.

The larva collected was subsequently sent for identification to the Parasitology Unit of the University of Foggia. Here it was cut into three parts; thoracic and terminal segments were subjected to microscopy, while the abdominal segment was used for molecular identification.

2.1. Microscopy. The larva portions were first macerated in lactophenol for one week at $45^{\circ} \mathrm{C}$ on a hot plate and then mounted on a Hoyer's-embedded slide. Observations, identification and light images were obtained using an Olympus BX51 light microscope (LM) equipped with an Olympus E330 camera.

The larva portions were then remounted from the slide and prepared for scanning electron microscopy (SEM). Hoyer's fluid was removed with water, and the larva portions were dehydrated using a graded ethanol series, dried with a Leica EM CPD300 critical point dryer, mounted on SEM stubs using conductive carbon adhesive tabs, and sputtercoated using an Edwards S150A sputter coater apparatus. A Hitachi TM3030 tabletop scanning electron microscope was used for observations and micrographs.

The III instar larva was identified using the key for myiasis-causing flies [15] and for the Sarcophagidae family to subgenus level [10]. The most distinctive characters available in literature for Sarcophaga (Liopygia) spp. identification, that is, Sarcophaga argyrostoma $[8,10,16,17]$, Sarcophaga crassipalpis [18], and Sarcophaga cultellata [3], were considered. In particular, the presence/absence of spines/warts on the $\mathrm{t} 2$ interband area, the number of lobes on the anterior spiracles, the shape of the posterior spiracles, and cephalo-skeleton were compared (Table 1).

2.2. Molecular Investigation. DNA was extracted using the Nucleospin Tissue kit (Macherey-Nagel, Netherlands) according to the manufacturer's instructions. The extracted DNA was eluted in $50 \mu \mathrm{L}$ of distilled water and the sample was stored at $-20^{\circ} \mathrm{C}$.
Universal primer pairs (F1/R1; F2/R2; F3/R3) (Table 2) [20] were used to amplify fragments of different sizes within the cytochrome oxidase subunit I gene. The PCR reaction mix contained 5x Phire Reaction Buffer (Thermo Scientific, USA), $200 \mu \mathrm{M}$ of dNTPs (Qiagen, USA), $10 \mu \mathrm{L}$ of each primer pairs, 1U Phire Hot Start II DNA Polymerase (Thermo Scientific, USA), and $1 \mu \mathrm{L}$ of genomic DNA in a final volume of $20 \mu \mathrm{L}$. The PCR protocol was as follows: denaturation at $98^{\circ} \mathrm{C}$ for $30 \mathrm{~s}$, followed by $98^{\circ} \mathrm{C}$ for $5 \mathrm{~s}$ and $59^{\circ} \mathrm{C}$ for $30 \mathrm{~s}$ for 35 cycles, and finally $72^{\circ} \mathrm{C}$ for $1 \mathrm{~min}$. A negative control (PCR water) was included in each PCR run. The PCR products were run on $1.2 \%$ agarose gel and positive samples were purified with Exonuclease I (EXO I) and Thermosensitive Alkaline Phosphatase (FAST AP) (Fermentas) enzymes.

Amplicons obtained were directly sequenced in both directions using the ABI PRIMS BygDye Terminator v. 3.1 Cycle Sequencing Kit (Applied Biosystems, Foster City, California, USA) with the same primers as the respective PCRs reaction, according to the manufacturer's instructions. Sequences obtained were determined on an ABI PRISM 3130 Genetic Analyzer (Applied Biosystems), electropherograms were inspected by eye, and consensus sequence was determined assembling different PCR fragments.

Our sequence was aligned with the available nucleotide sequences in publicly databases, using the BLASTn software and the Muscle application implemented in SeaView 4. Moreover, our sequence was aligned with all the S. argyrostoma DNA barcode sequences [ID numbers: NICC0085, accession number: JQ582081; NICC0637, accession number: JQ582123] [21] using Bioedit V.5 software and the Muscle application implemented in SeaView 4. Phylogenetic analyses were conducted in MEGA6 using the Neighbor-Joining method. The evolutionary distances were computed using the p-distance method and were in the units of the number of base differences per site. The analysis involved 45 nucleotide sequences. Codon positions included were $1 s t+2 n d+3 r d+$ Noncoding. All positions containing gaps and missing data were eliminated. There were 61 positions in the final dataset. The tree was rooted using a Wohlfahrtia magnifica sequence as an outgroup (accession number: FJ379616).

\section{Results}

3.1. Microscopy. The larva examined in this study had the following characters:

(1) No spines on $\mathrm{t} 2$ interband area (Figures 1(a) and 1(b))

(2) Anterior spiracles with 14 lobes (Figure 1(c))

(3) Kidney-shaped posterior spiracles (Figure 2)

(4) Short ventral arch of the posterior spiracle, not reaching the inner arch area (Figure 2)

(5) Ventral cornua of the cephaloskeleton with developed window (Figure 3(a))

(6) Dorsal cornua not clearly 8-shaped (Figure 3(a)) and tip shape of the intermediate sclerite apparently truncated (Figure 3(c))

(7) Spiracular cavity with very wrinkled edge (Figure 4) 


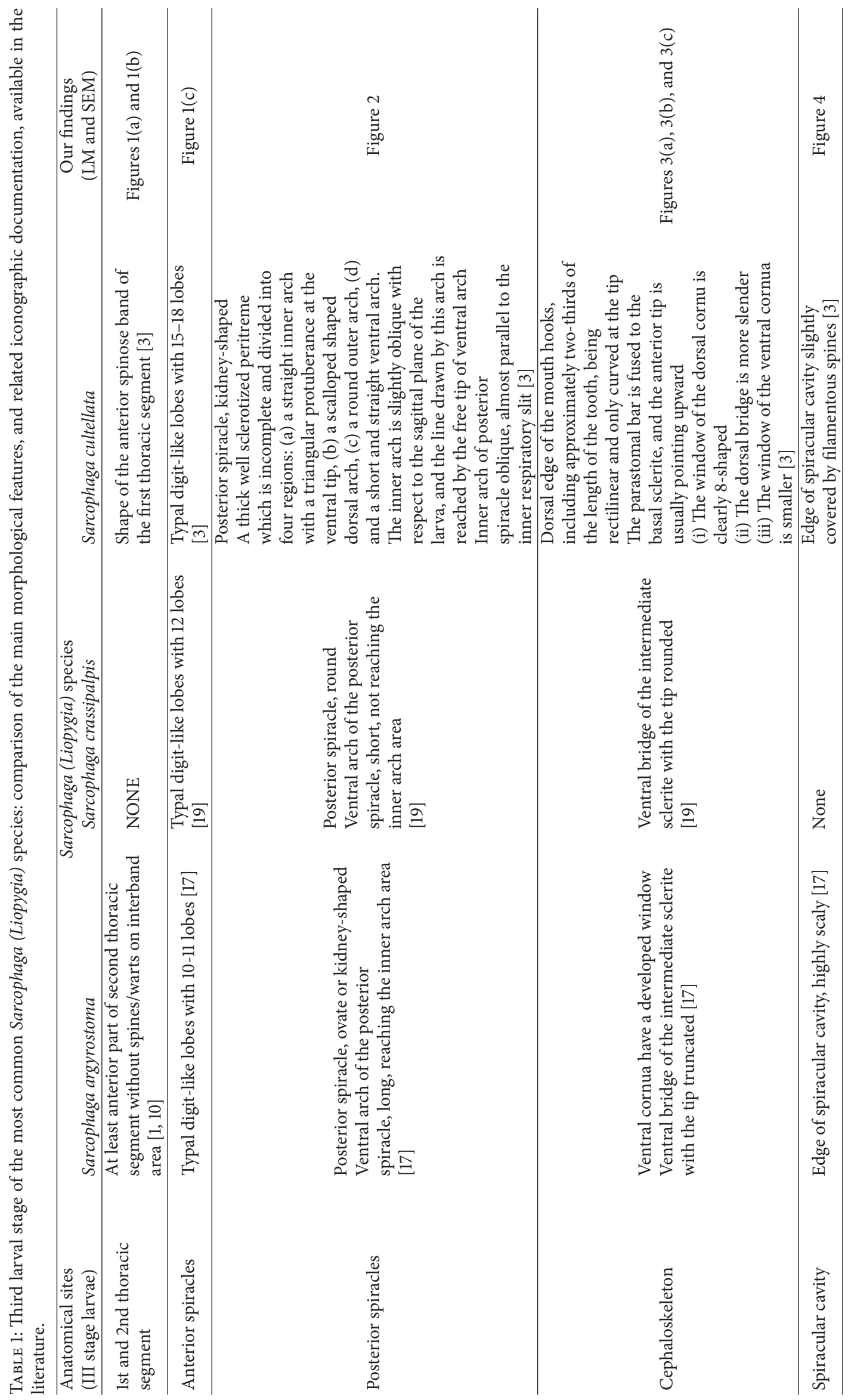


TABLE 2: Universal primer sequences within the mitochondrial cytochrome oxidase gene subunit I (coxI) (by Kim et al., [20]).

\begin{tabular}{lrr}
\hline Name & Sequence & Binding site \\
\hline F1 & CCTTTAGAATTGCAGTCTAATGTCA & tRNA-cysteine \\
F2 & GGAGGATTTGGAAATTGATTAGTTCC & $220-245$ on COI \\
F3 & CTGCTACTTTATGAGCTTTAGG & $2-23$ on COI \\
R1 & CCTAAATTTGCTCATGTTGACA & $1000-1022$ on COI \\
R2 & CAAGTTGTGTAAGCATC & $1327-1343$ on COI \\
R3 & CCAAAGAATCAAAATAAATGTTG & $688-710$ on COI \\
\hline
\end{tabular}

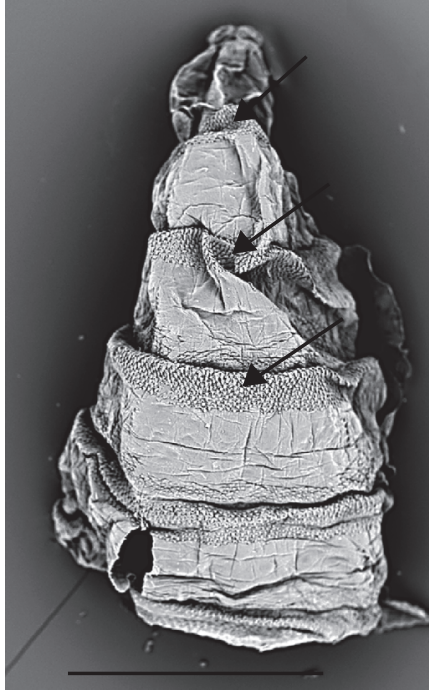

(a)

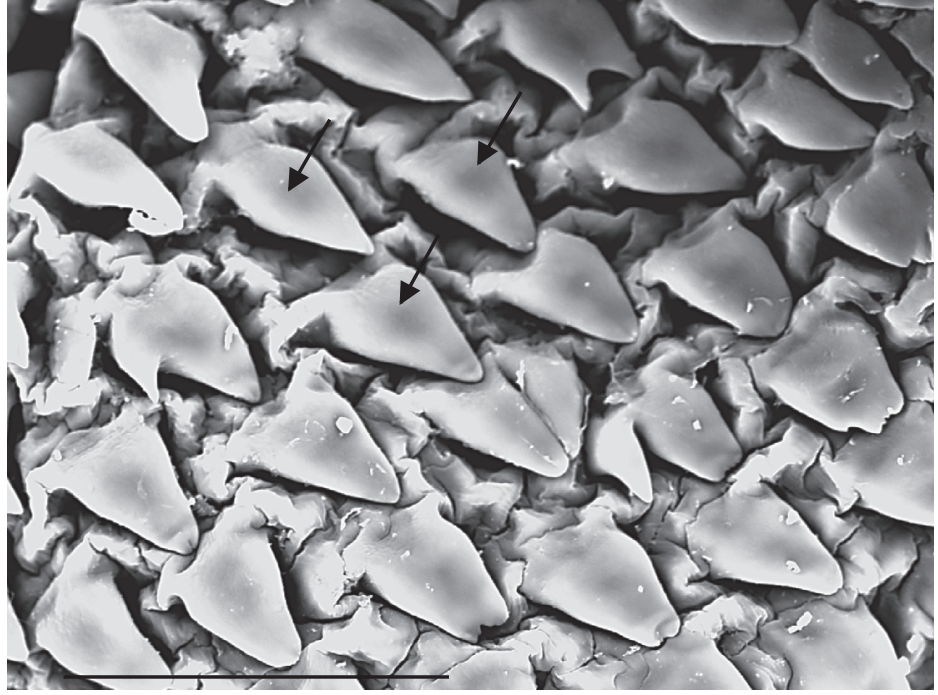

(b)

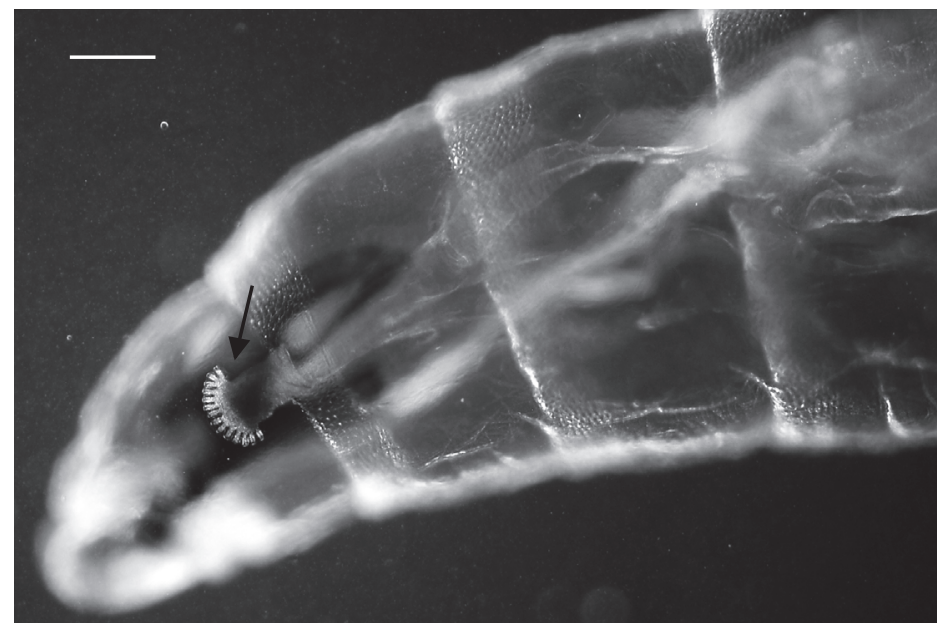

(c)

FIgure 1: Sarcophaga sp. third instar larva. (a) SEM image, anterior dorsal view of the body showing the interband areas free of any spines/warts and the anterior spinose band (arrows). (b) SEM image, detail of the spines on the anterior spinose band areas (arrows). (c) LM image, lateral view of anterior body showing anterior spiracle with 14-digit-like lobes clearly visible. Scale bar: $1 \mathrm{~mm}(\mathrm{a}) ; 50 \mu \mathrm{m}(\mathrm{b})$; $100 \mu \mathrm{m}(\mathrm{c})$.

According to the comparative table and illustrations (Table 1), of the seven features above, one (number 5) and apparently another (number 6) point to S. argyrostoma species; one character (number 1) points to all three species (S. argyrostoma and/or S. cultellata and/or S. crassipalpis); one character could be related to both S. argyrostoma and/or S. cultellata (number
3), one to both $S$. crassipalpis and/or S. cultellata species (number 4), and two features (nos. 2 and 7) are apparently not associated with any of the three species.

3.2. Molecular Investigations. Sequencing provided a good quality sequence of about $735 \mathrm{bp}$. BLASTn analysis (https://blast 


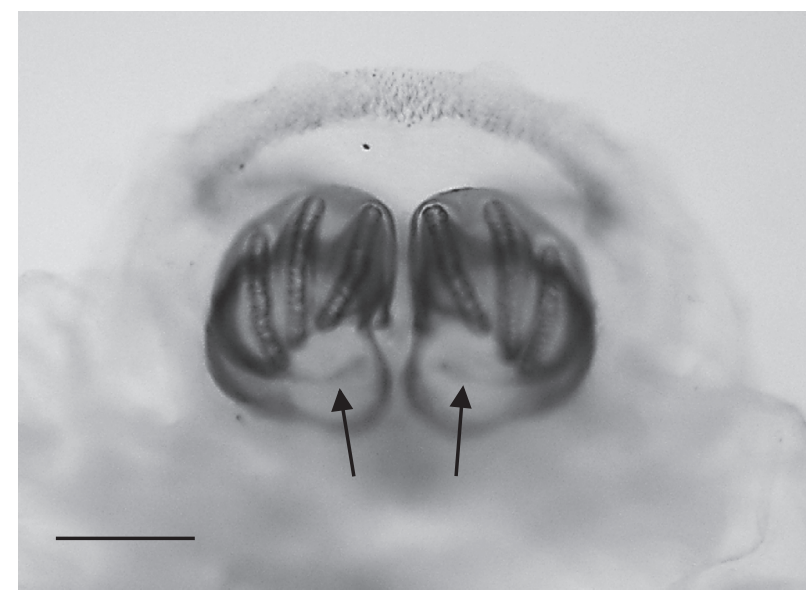

Figure 2: Sarcophaga spp. third instar larva: LM image, overview of kidney-shaped posterior spiracles with ventral arches (arrows) not reaching the inner arch area. Scale bar: $100 \mu \mathrm{m}$.

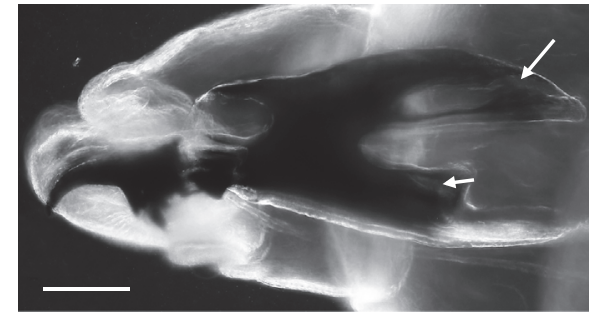

(a)

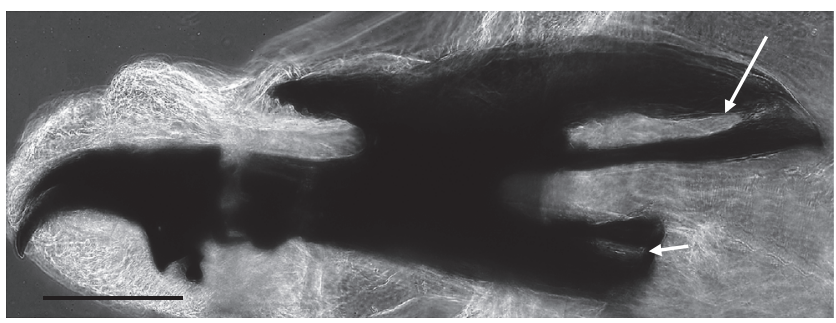

(b)

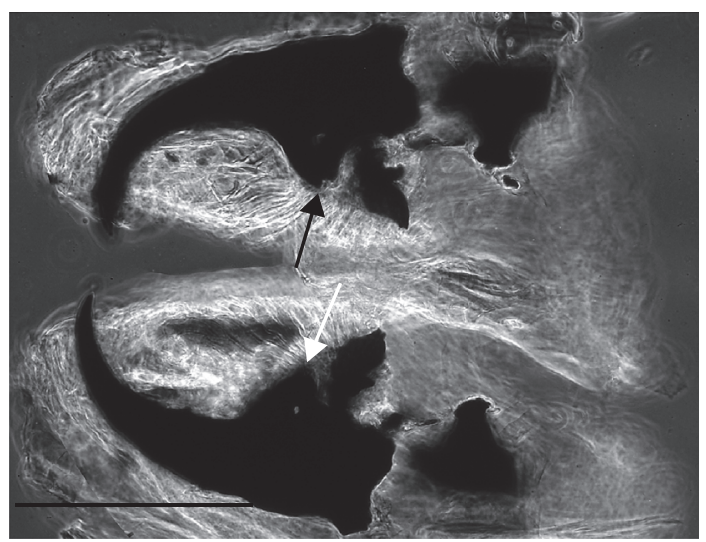

(c)

FIGURE 3: Sarcophaga third instar larva: LM image: (a) cephaloskeleton with dorsal cornua showing window apparently 8-shaped (arrow) while the ventral cornua window is smaller but still well developed (arrowhead). (b) Same sample showing a different shape (more elongated and not 8 -shaped) of the window due to a different orientation of the sample in the slide. (c) Detail of the ventral bridge of the intermediate sclerite with truncated tip (arrows). Scale bar: $100 \mu \mathrm{m}$.

.ncbi.nlm.nih.gov/Blast.cgi) showed that our sequence matched the target GenBank coxI sequence S. argyrostoma with a $99 \%$ of identity. Ninety-nine percent of identity was also obtained by alignment with coxI DNA barcode region sequence [21]. The sequence was deposited in GenBank under accession number KU570766. The phylogenetic analysis showed that the Italian sequence clusters with the other S. argyrostoma sequences (from Belgium, France,
Egypt, and USA) in a monophyletic group with a high bootstrap value (>95) and that this clade is distant from the other Sarcophaga species (Figure 5).

\section{Discussion}

In this study, the single III instar larva showed some characters that are typical for S. argyrostoma, whereas some other 


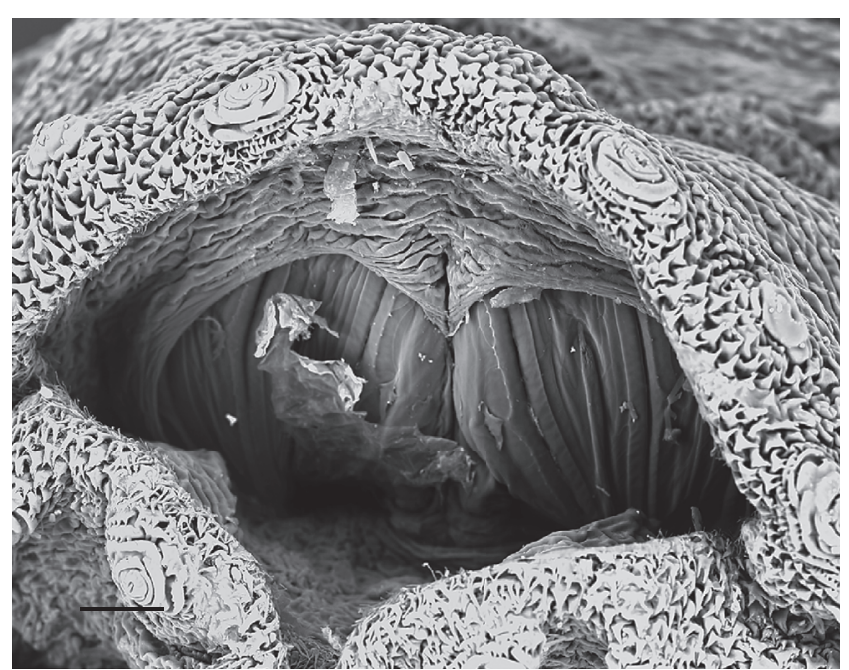

(a)

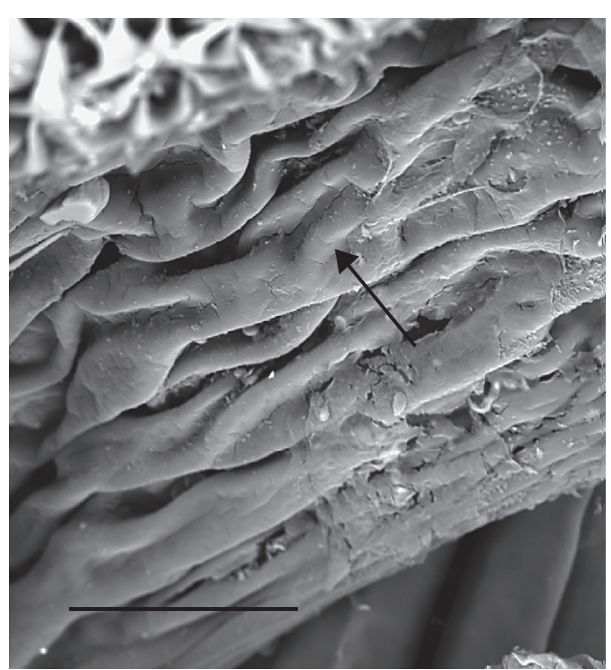

(b)

FIgURE 4: Sarcophaga sp. third instar larva: SEM images. (a) Overview of the posterior spiracle cavity. (b) Detail showing highly wrinkled edge of the posterior cavity (arrow). Scale bar: $100 \mu \mathrm{m}(\mathrm{a}) ; 30 \mu \mathrm{m}(\mathrm{b})$.

characters were not. Thus, according to the morphological characters it was only possible to assume the identification of the single III instar larva as Sarcophaga (Liopygia) argyrostoma but a molecular investigation analysis was needed for confirmation.

In addition to Sarcophaga (Liopygia) argyrostoma, two other species in the same subgenus are found in Mediterranean countries: $S$. (L.) crassipalpis Macquart, 1839, and $S$. (L.) cultellata Pandelle, $1986[3,10]$. These three species have been recorded either in case reports of wound myiasis in live humans or in supporting forensic investigations of human corpses. In Southern Italy, S. crassipalpis was identified in a corpse [18], while S. cultellata has been identified in human corpses in Spain [3,22] and also in Northern Italy [23].

The main key morphological characters for differentiation of the III larval stage in these three species of the subgenus Liopygia involve dorsal surface of the II thoracic segment, anterior spiracle digits/papillae, posterior spiracles, and the shape of the cephaloskeleton $[3,10]$. In our case, those characters do not appear clearly unequivocal: some key features seem related to all three species, that is, the second thoracic segment interband area [10], and shape of the posterior spiracles $[3,17,18]$, while some characteristics cannot be ascribed to any of these three species, that is, the number of lobes on anterior spiracles and edge of the posterior spiracular cavity $[3,17]$ (Table 1 ). Moreover, some characters are difficult to read, since their appearance and shape may change with the position of the sample on the slide. An obvious example is provided by the shape of the dorsal cornua window, which appears different on the same sample, merely because of its position (please compare Figures 3(a) and 3(b)). Generally, the main obstacle in the use of morphological markers for identification of larvae of subgenus Liopygia is lack of solid comparative analysis. Studies of potentially useful characters, based on a long series of individuals and unified methodology, should solve this problematic situation as it was done for larval stages of some other difficult taxa (e.g., [24, 25]).

Hence, when larvae come from the Liopygia distribution area, that is, the Mediterranean countries, the available morphological features may not be clear enough for comparison and correct identification. This problem, coupled with the difficulty of checking all characters when a single specimen is provided, calls for the use of molecular tools.

The robustness of the coxI gene as a diagnostic marker to lay the groundwork for identification of these three species of Sarcophaga has been demonstrated [21, 26]. Sarcophaga argyrostoma has been identified using a coxI sequence in an elderly patient with an external ophthalmomyiasis (with 97\% identity) [6] and very recently in a tracheotomized child's surgical wound (with an identity of $100 \%$ ) [8]. In the present study, the involvement of S. argyrostoma in the wound myiasis is confirmed with $99 \%$ identity.

This is the third case in Europe [4, 8]. Cases of myiasis caused by Sarcophaga species are apparently few, but it must be underlined that cases of myiasis often remain unrecorded or that, more frequently, identification is partial, that is, at genus level [27-31].

\section{Conclusion}

In conclusion, it is very important to record and correctly identify the species involved in nosocomial myiasis, particularly if patients are moved from geriatric homes to hospital for such problem and thus even become a legal issue.

Correct identification of the myiasis agent provides more detailed information on the responsibility that health units (hospitals, geriatric homes, etc.) must have towards patients. In this view, hygiene, protection from flies by physical barriers (screens or closed windows), efficient waste disposal measures to reduce the smell of decomposition, and insecticide 


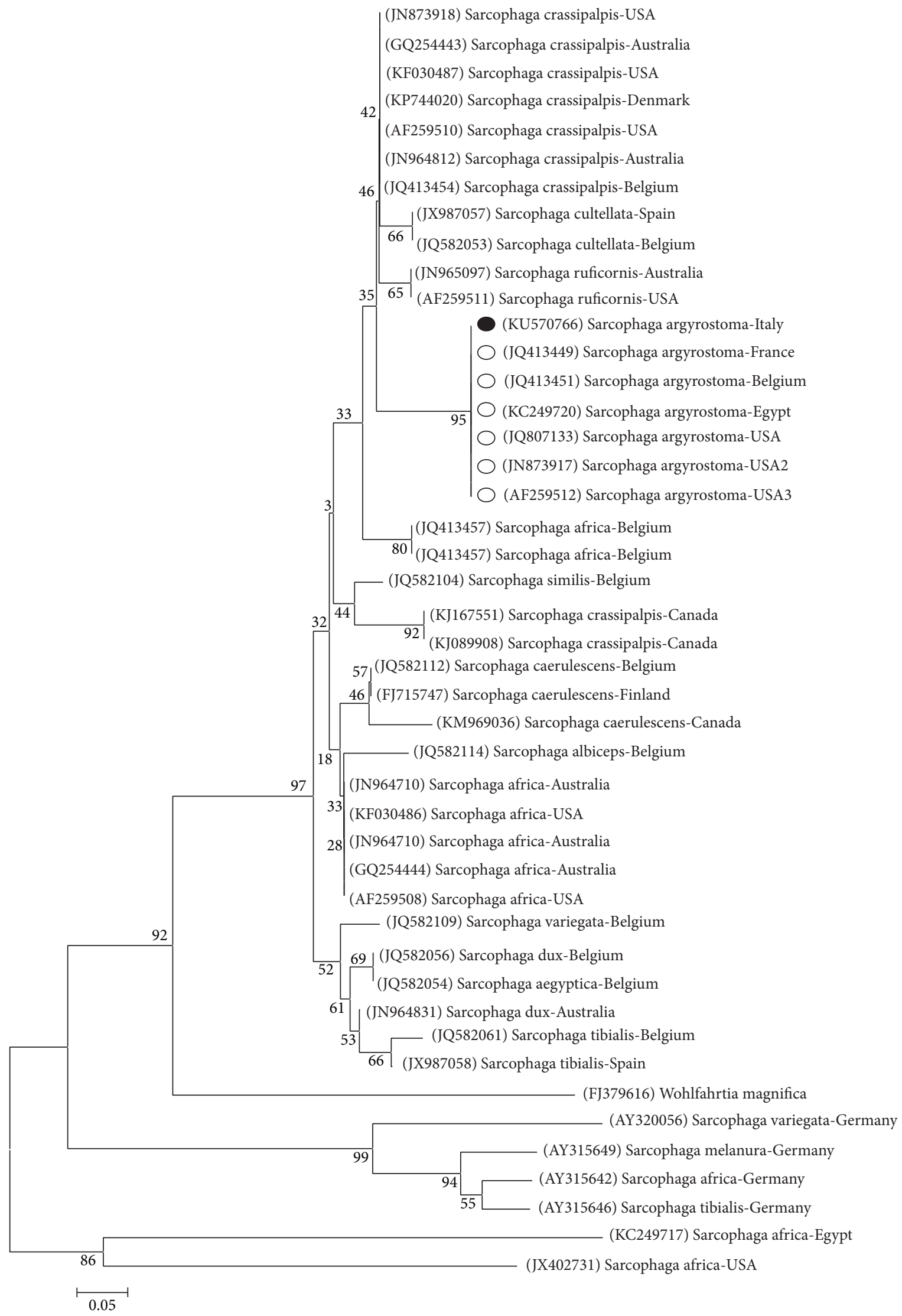

FIgURE 5: The Neighbor-Joining (NJ) phylogenetic tree based on analysis of the partial cytochrome $c$ oxidase subunit I gene of Sarcophaga species of forensic interest. The Sarcophaga argyrostoma sequence from the present study (black spot), 11 sequences representing the other Sarcophaga species belonging to Liopygia subgenus, that is, S. crassipalpis (9) and S. cultellata (2) and 20 other Sarcophaga species from GenBank were included in the analysis for comparative purposes. Wohlfahrtia magnifica was used as an outgroup. GenBank accession numbers (in brackets), Sarcophaga species, and country of origin are reported. 
sprays are basic prevention measures that hospitals should take.

\section{Competing Interests}

The authors declare that they have no competing interests.

\section{Authors' Contributions}

Annunziata Giangaspero and Antonella Di Palma contributed equally to the work.

\section{Acknowledgments}

The authors wish to thank Dr. Giulia Sartoni from Bufalini Hospital (Geriatric Unit) for her collaboration. The present work was partially funded by L.A.I.F.F. Project (Code no. 47); PO Puglia FESR-2007-2013, Asse I, Linea 1.2. Accordo di Programma Quadro in Materia di Ricerca Scientifica. Intervento "Reti di Laboratori Pubblici di Ricerca" (responsible: Annunziata Giangaspero), and financially supported by the Polish National Science Centre (Grant no. 2012/07/B/NZ8/00158) (responsible: Krzysztof Szpila).

\section{References}

[1] K. Szpila, A. Mądra, M. Jarmusz, and S. Matuszewski, "Flesh flies (Diptera: Sarcophagidae) colonising large carcasses in Central Europe," Parasitology Research, vol. 114, no. 6, pp. 23412348, 2015.

[2] R. Richet, R. M. Blackith, and T. Pape, Sarcophaga of France (Diptera: Sarcophagidae), Pensoft Series Faunistica, Pensoft, Sofia, Bulgaria, 2011.

[3] N. Ubero-Pascal, Á. Paños, M.-D. García, J.-J. Presa, B. Torres, and M.-I. Arnaldos, "Micromorphology of immature stages of Sarcophaga (Liopygia) cultellata Pandellé, 1896 (Diptera: Sarcophagidae), a forensically important fly," Microscopy Research and Technique, vol. 78, no. 2, pp. 148-172, 2015.

[4] I. Burgess and P. D. R. Spraggs, "Myiasis due to Parasarcophaga argyrostoma - first recorded case in Britain," Clinical and Experimental Dermatology, vol. 17, no. 4, pp. 261-263, 1992.

[5] S. Uni, S. Shinonaga, Y. Nishio et al., "Ophthalmomyiasis caused by Sarcophaga crassipalpis (Diptera: Sarcophagidae) in a hospital patient," Journal of Medical Entomology, vol. 36, no. 6, pp. 906-908, 1999.

[6] S. Graffi, A. Peretz, A. Wilamowski, H. Schnur, F. Akad, and M. Naftali, "External ophthalmomyiasis caused by a rare infesting larva, Sarcophaga argyrostoma," Case Reports in Ophthalmological Medicine, vol. 2013, Article ID 850865, 2 pages, 2013.

[7] H. Hiraoka, T. Ozawa, J. Sowa-Osako et al., "Repeated myiasis in a female vulvar squamous cell carcinoma caused by Lucilia sericata and Sarcophaga crassipalpis," Journal of Dermatology, vol. 42, no. 8, pp. 840-841, 2015.

[8] F. Severini, E. Nocita, and F. Tosini, "Myiasis of the tracheostomy wound caused by sarcophaga (Liopygia) argyrostoma (diptera: sarcophagidae): molecular identification based on the mitochondrial cytochrome c oxidase I gene," Journal of Medical Entomology, vol. 52, no. 6, pp. 1357-1360, 2015.

[9] F. Francesconi and O. Lupi, "Myiasis," Clinical Microbiology Reviews, vol. 25, no. 1, pp. 79-105, 2012.
[10] K. Szpila, R. Richet, and T. Pape, “Third instar larvae of flesh flies (Diptera: Sarcophagidae) of forensic importance-critical review of characters and key for European species," Parasitology Research, vol. 114, no. 6, pp. 2279-2289, 2015.

[11] M. Giroux, T. Pape, and T. A. Wheeler, “Towards a phylogeny of the flesh flies (Diptera: Sarcophagidae): morphology and phylogenetic implications of the Acrophallus in the subfamily Sarcophaginae," Zoological Journal of the Linnean Society, vol. 158 , no. 4, pp. 740-778, 2010.

[12] K. Saigusa, M. Takamiya, and Y. Aoki, "Species identification of the forensically important flies in Iwate prefecture, Japan based on mitochondrial cytochrome oxidase gene subunit I (COI) sequences," Legal Medicine, vol. 7, no. 3, pp. 175-178, 2005.

[13] S. H. Tan, M. Rizman-Idid, E. Mohd-Aris, H. Kurahashi, and Z. Mohamed, "DNA-based characterisation and classification of forensically important flesh flies (Diptera: Sarcophagidae) in Malaysia," Forensic Science International, vol. 199, no. 1-3, pp. 43-49, 2010.

[14] K. A. Meiklejohn, J. F. Wallman, and M. Dowton, "DNA-based identification of forensically important Australian Sarcophagidae (Diptera)," International Journal of Legal Medicine, vol. 125, no. 1, pp. 27-32, 2011.

[15] M. A. Taylor, R. L. Coop, and R. L. Wall, Veterinary Parasitology, Blackwell, 2008.

[16] A. Awad, S. Abdel-Salam, R. Abou El-Ela, A. Abdel-Aal, and D. Mohamed, "Ultrastructure comparison of the sensory morphology of the first- and third-instar larvae of Parasarcophaga argyrostoma (Robineau-Desvoidy) (Diptera: Sarcophagidae)," Egyptian Journal of Biology, vol. 5, pp. 148-154, 2003.

[17] A. Draber-Mońko, T. Malewski, J. Pomorski, M. Łoś, and P. Ślipiński, "On the morphology and mitochondrial DNA barcoding of the flesh fly Sarcophaga (Liopygia) argyrostoma (Robineau-Desvoidy, 1830) (Diptera: Sarcophagidae)—an important species in forensic entomology," Annales Zoologici, vol. 59, no. 4, pp. 465-493, 2009.

[18] T. Bonacci, V. Vercillo, P. Brandmayr, A. Fonti, C. Tersaruolo, and T. Z. Brandmayr, "A case of Calliphora vicina RobineauDesvoidy, 1830 (Diptera, Calliphoridae) breeding in a human corpse in Calabria (southern Italy)," Legal Medicine, vol. 11, no. 1, pp. 30-32, 2009.

[19] T. Bonacci, S. Greco, B. Cavalcanti, P. Brandmayr, and V. Vercillo, "The flesh fly Sarcophaga (Liopygia) crassipalpis Macquart 1839 as an invader of a corpse in Calabria (Southern Italy)," Journal of Forensic Science \& Criminology, vol. 2, no. 1, p. 104, 2014.

[20] Y.-H. Kim, S. E. Shin, C. S. Ham et al., "Molecular identification of necrophagous muscidae and sarcophagidae fly species collected in Korea by mitochondrial cytochrome C oxidase subunit I nucleotide sequences," The Scientific World Journal, vol. 2014, Article ID 275085, 9 pages, 2014.

[21] K. Jordaens, G. Sonet, R. Richet, E. Dupont, Y. Braet, and S. Desmyter, "Identification of forensically important Sarcophaga species (Diptera: Sarcophagidae) using the mitochondrial COI gene," International Journal of Legal Medicine, vol. 127, no. 2, pp. 491-504, 2013.

[22] Y. Velásquez, C. Magaña, A. Martínez-Sánchez, and S. Rojo, "Diptera of forensic importance in the Iberian Peninsula: larval identification key," Medical and Veterinary Entomology, vol. 24, no. 3, pp. 293-308, 2010.

[23] T. Pape, "Catalogue of the Sarcophagidae of the world (Insecta: Diptera)," Memoirs on Entomology International, vol. 8, p. 558, 1996. 
[24] K. Szpila, M. J. R. Hall, T. Pape, and A. Grzywacz, "Morphology and identification of first instars of the European and Mediterranean blowflies of forensic importance. Part II. Luciliinae," Medical and Veterinary Entomology, vol. 27, no. 4, pp. 349-366, 2013.

[25] A. Grzywacz, M. J. R. Hall, and T. Pape, "Morphology successfully separates third instar larvae of Muscina," Medical and Veterinary Entomology, vol. 29, no. 3, pp. 314-329, 2015.

[26] N. Bajpai and R. R. Tewari, "Mitochondrial DNA sequencebased phylogenetic relationship among flesh flies of the genus Sarcophaga (Sarcophagidae: Diptera)," Journal of Genetics, vol. 89, no. 1, pp. 51-54, 2010.

[27] O. S. Aldemir, E. Şimşek, and A. Ayan, "The first case of otomyiasis caused by Sarcophaga spp. (Diptera; Sarcophagidae) larvae in a goose in the world," Türkiye Parazitoloji Dergisi, vol. 38, no. 3, pp. 211-213, 2014.

[28] F. D. Kaya, O. Orkun, A. Çakmak, A. Ç. Inkaya, and S. Ergüven, "Cutanous myiasis caused by Sarcophagaspp. larvae in a diabetic patient," Mikrobiyoloji Bulteni, vol. 48, no. 2, pp. 356-361, 2014.

[29] M. Dutto and M. Bertero, "Cutaneous superficial myiasis: report of a rare nosocomial parasitic disease caused by Sarcophaga spp. (Diptera, Sarcophagidae)," Central European Journal of Public Health, vol. 19, no. 4, pp. 232-234, 2011.

[30] H. Dagci, F. Zeyrek, Y. K. Gerzile, S. B. Sahin, S. Yagci, and A. Uner, "A case of myiasis in a patient with psoriasis from Turkey," Parasitology International, vol. 57, no. 2, pp. 239-241, 2008.

[31] M. Türk, I. Afşar, Y. Ozbel, A. G. Sener, A. Uner, and M. Türker, "A case of nasomyiasis whose agent was Sarcophaga sp," Turkiye Parazitol Derg, vol. 30, no. 4, pp. 330-332, 2006. 

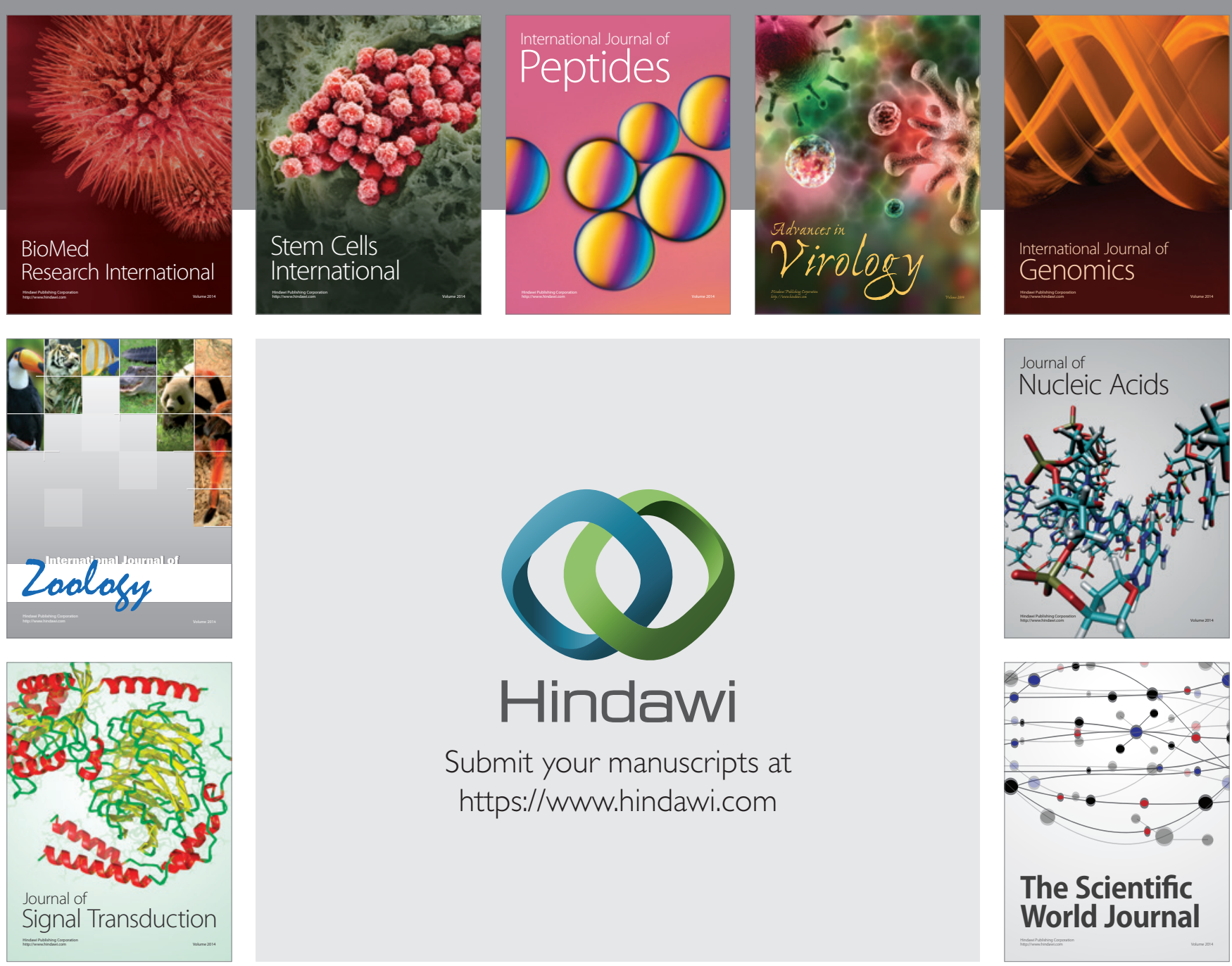

Submit your manuscripts at

https://www.hindawi.com
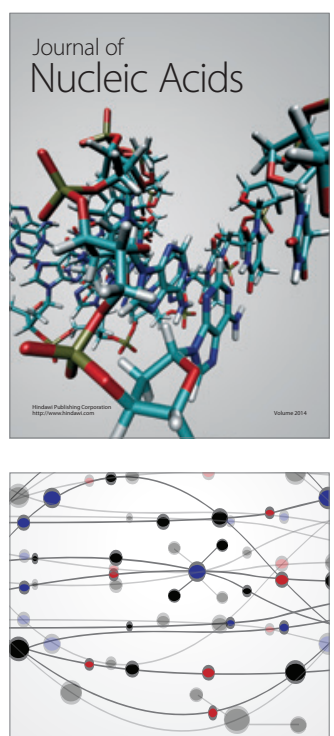

The Scientific World Journal
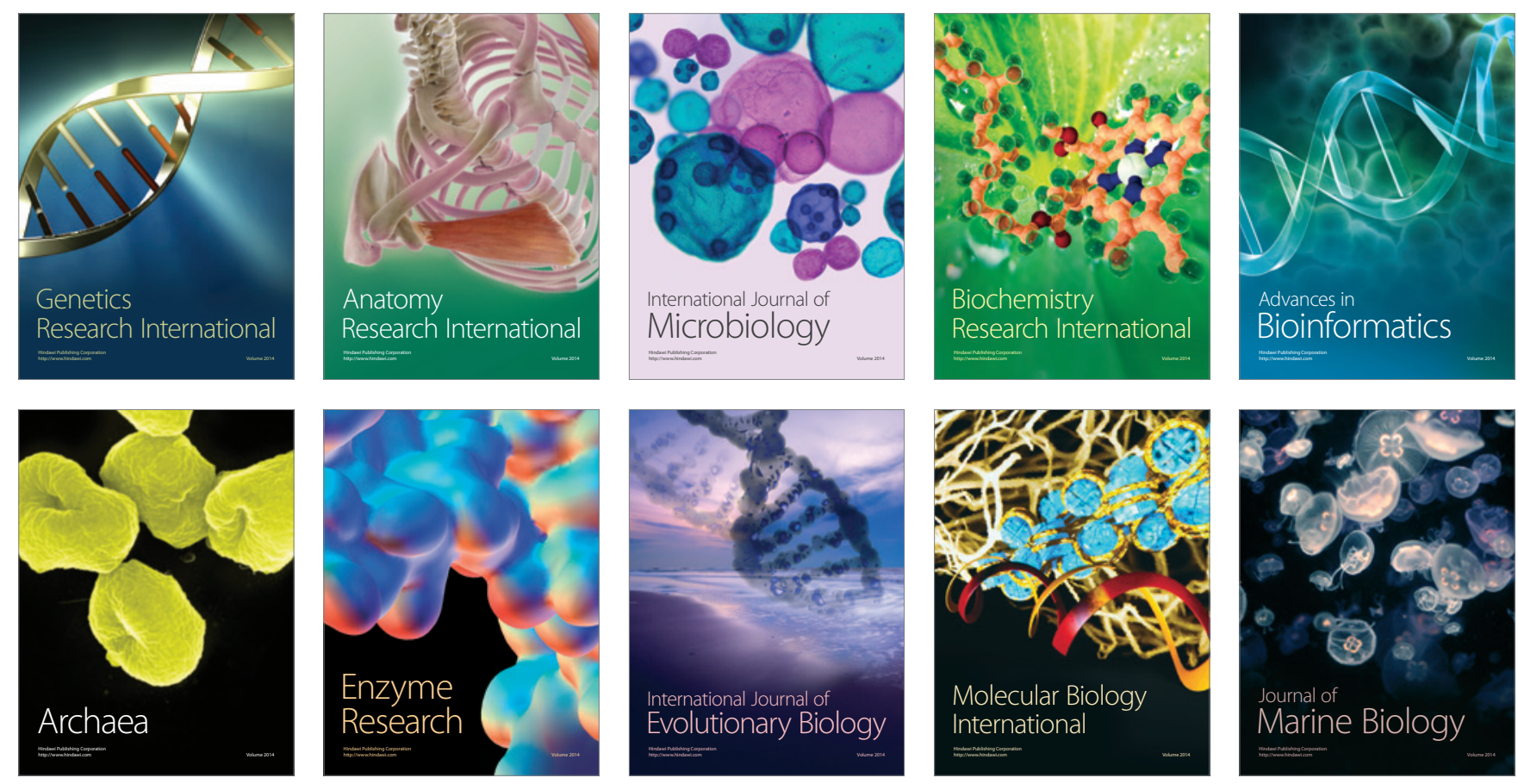